\title{
MAXILLARY AND MANDIBULAR CANINES' TORQUE EXPRESSION DURING RETRACTION USING 0.022" ROTH PRESCRIPTION BRACKETS
}

\author{
Fouad A. El Sharaby", Haya A. Barsoum ${ }^{* *}$ and Hend S. ElSayed ${ }^{* * *}$
}

\begin{abstract}
Background: In extraction cases, canine torque is of primary importance. Failure to maintain the root within the cancellous bone predisposes to root resorption and periodontal breakdown. Positive torque in the bracket or wire may prevent the proximity of the root and cortical bone as well as the subsequent side effects.

Objective: The aim of this study was to evaluate the torque control provided by 0.022 " Roth prescription brackets during canine retraction with sliding mechanics.

Materials and methods: Twenty-one patients $(19.2 \pm 2.7$ years $)$ with various malocclusions involving canine retraction were included in the trial. Cone beam computer tomography was acquired before and six months after the start of canine retraction. The change in canine torque was calculated from the pre- and post-retraction 3-dimensional images. Torque was measured for maxillary and mandibular canines as the angle between the canine's long axis and the mid-sagittal plane. The paired t-test was used to evaluate the change in torque.

Results: Statistically significant increase in the angle occurred for the maxillary $\left(3.088 \pm 5.627^{\circ}\right)$ and mandibular $\left(2.971 \pm 5.427^{\circ}\right)$ canines.

Conclusion: Canines could express labial crown inclination during retraction despite the absence of a positive built-in bracket torque.
\end{abstract}

KEYWORDS: Canine retraction, bracket torque, wire torsion, root resorption, fenestration.

* Associate Professor, Department of Orthodontics and Dentofacial Orthopedics, Faculty of Dentistry, Cairo University, Cairo, Egypt.

** Resident, Department of Orthodontics and Dentofacial Orthopedics, Faculty of Dentistry, Future University in Egypt, Cairo, Egypt

*** Associate Professor, Department of Orthodontics \& Pediatric Dentistry, Oral and Dental Research Division, National Research Centre, Giza, Egypt \& Adjunct Assistant Professor, Department of Epidemiology \& Health Promotion, College of Dentistry, NYU. 


\section{INTRODUCTION}

Controlled mechanotherapy of canine retraction provides efficient tooth movement and proper tooth position as well as limits side effects. Torque control is of particular interest when retracting the canines. Careful planning is required to move the wide bucco-lingual dimension of the canine root through the cancellous bone. Excessive contact between the roots and the cortical plates of the alveolus may lead to excessive root resorption. Bucco-lingual root movement ${ }^{[1]}$ may create alveolar bone dehiscence, fenestration, and gingival recession. Torque in patients with thin cortical bone, buccally displaced canines, old extraction sites, and extraction cases require special consideration..$^{[2]}$

Therefore, it has been recommended to use bracket prescriptions with a positive torque to bring the roots within the alveolus during canine retraction. ${ }^{[3]}$ However, torque increases the contact between the bracket slot and the arch wires generating more friction during sliding mechanics. ${ }^{[4,5]}$

The aim of this study was to evaluate if the 0.022 " Roth prescription brackets would provide adequate torque control during sliding canine retraction.

\section{METHODOLOGY}

This was a before and after clinical trial conducted at FUE between January 2018 and October 2019. The university's ethical committee reviewed and approved the protocol in April 2017.

Twenty-one patients (females; 13 and males; 8) were recruited from the University Orthodontic Department. The patients' average age was 19.2 \pm 2.7 years. Patients with severe dental crowding, bimaxillary dentoalveolar protrusion, Class II or Class III (Angles' classification), and indicated for canine retraction were eligible for the trial. Patients were excluded if they had received previous orthodontic treatment, suffered from periodontal disease, periodontal pockets, or gingival recession. Other exclusion criteria were medications that may affect bone remodeling and tooth movement. Pregnant women or those planning to get pregnant were excluded since cone beam computer tomography was planned for the outcome assessment. Willing participants signed informed consents before joining the trial.

At the start of treatment, all patients were referred for cone beam computer tomography (CBCT) (Acteon X-mind Trium CBCT machine, La Ciotat, France) following the ALARA guideline. ${ }^{\left[{ }^{[6}\right.}$ According to the user's manual, a minimum field of view $(80 \times 80 \mathrm{~mm})$ and a voxel size of $0.15 \mathrm{~mm}$ were used. ${ }^{[7]}$

The first molars were banded in all the patients and the canines and premolars were bonded using a 0.022" Roth brackets (Mini Master; American Orthodontics, Sheboygan, Wis.). The canine bracket torque prescription was -2 and -11 for the upper and lower canines, respectively. After leveling and alignment of the posterior segment, the canine retraction was started with $9 \mathrm{~mm}$ closed Nickel titanium coil springs over 0.016 "X0.022" stainless steel arch wires. The coil springs applied $150 \mathrm{gm}$ and were reactivated every 6 weeks so that the force was re-established. After six months of canine retraction, a second CBCT was taken with the same parameters as the pre-retraction exposure.

The raw DICOM images were imported to InVivoDental 5 software which constructed the 3-dimensional images. The mid-sagittal plane was constructed. It extended between the anterior nasal spine, posterior nasal spine and incisive foramen. (Fig. 1)

The outcome of interest was the canine torque which was measured as the angle between the midsagittal plane and the canine long axis, drawn from cusp tip to root apex. (Fig. 1) The canine torque change was calculated as the difference between the pre- and post-retraction angle. 


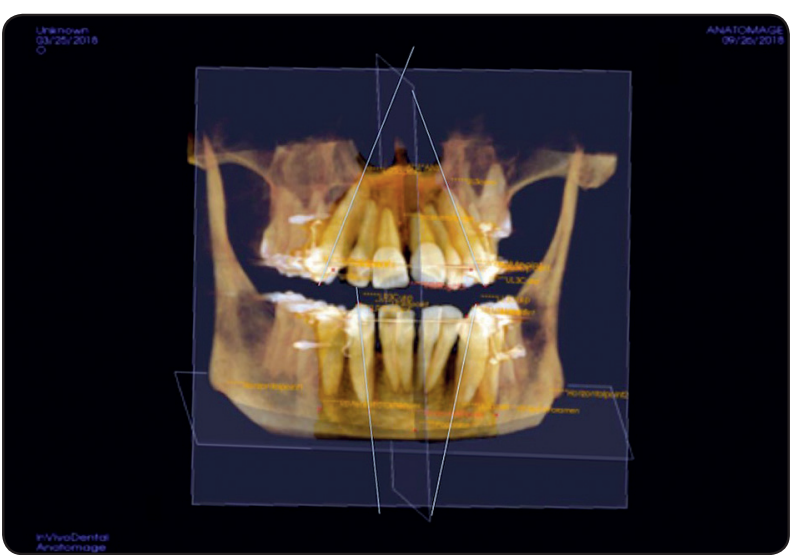

Fig. (1): Canine torque measured as the angle between the long axis of the canine and the mid-sagittal plane

Canine torque was measured by a second outcome assessor for ten CBCTs and the inter-rater agreement was tested.

\section{Statistical analysis}

The Dahlberg's error was calculated. The interobserver reliability was evaluated using the Interclass Correlation Coefficient (ICC). The normality of the data was tested using a distribution histogram and the Shapiro-Wilck test. The paired t-test was used to compare the pre- and post-retraction root torque. All tests were two-tailed and the confidence level was set at $95 \%$.

\section{RESULTS}

The ICC (0.97) showed excellent inter-rater agreement. Dahlberg's error was 0.09. The data showed a normal distribution. The mean difference of canine torque showed a significant increase for both the maxillary and mandibular canines. (Table 1) The $95 \%$ confidence interval for the maxillary and mandibular canine torque was $1.138-5.038$ and 0.961 - 4.981, respectively.

\section{DISCUSSION}

The force distribution in the periodontal ligament, when a torque movement is intended, is concentrated at the root apex and the alveolar crest. ${ }^{[8]}$ This stress pattern has been found as a risk factor for excessive root and alveolar crest resorption. ${ }^{[9]}$ The proper torque can be applied through the bracket prescriptions and wire bending. During canine retraction, a positive torque bracket prescription may aid in moving the roots through the cancellous bone without the need for wire bending. Several prescriptions are available, specifically for extraction cases where a neutral or positive torque may be preferred. ${ }^{[10]}$

Brackets and wire combinations show that bracket torque expression is greatly decreased in clinical practice. ${ }^{[1]}$ Rectangular arch wires were more efficient for torque expression than square wires in 0.018 and 0.022 Slot brackets. ${ }^{[12]}$

A systematic review found that the engagement angle between the wire and slot was larger and more variable than that reported by manufacturers. The arch wire edge shape and dimension as well as the slot dimension affected the torque expression. ${ }^{[13]}$ In our study, the 0.022 " Roth bracket was combined with 0.016 "X0.022" stainless steel archwire for canine retraction to facilitate sliding.

TABLE (1): The descriptive statistics and the change in torque for the maxillary and mandibular canines

\begin{tabular}{llccccc}
\hline & & $\begin{array}{c}\text { Before retraction } \\
\text { Mean } \pm \text { SD }\end{array}$ & $\begin{array}{c}\text { After retraction } \\
\text { Mean } \pm \text { SD }\end{array}$ & $\begin{array}{c}\text { Mean Difference } \\
\pm \text { SD }\end{array}$ & t-value & p-value \\
\hline \multirow{2}{*}{ Canine torque } & Maxillary $\left({ }^{\circ}\right)$ & $7.96 \pm 3.94$ & $11.05 \pm 5.32$ & $3.088 \pm 5.627$ & 3.1044 & $0.0041^{*}$ \\
\cline { 2 - 7 } & Mandibular $\left(^{\circ}\right)$ & $8.073 \pm 3.89$ & $11.04 \pm 5.55$ & $2.971 \pm 5.427$ & 2.8966 & $0.0074 *$ \\
\hline
\end{tabular}

SD: standard deviation, *: statistical significance: $p<0.05$ 
The Roth prescription was used to promote a functional occlusion with the preservation of the periodontium and the temporomandibular joint. ${ }^{[14]}$

Since the vertical position of the bracket greatly affects the torque expression, the brackets were placed in the middle of the crown. ${ }^{[15]}$ It was assumed that the clinical crown was a good indicator for bracket positioning as the patients had no gingival recession, hypertrophy or significant alveolar bone loss.

The maxillary and mandibular canines showed labial crown torque despite the negative torque prescription of the Roth brackets. This may have been due to the retraction of the canines into a wider region of the arch and the insufficient expression of the torque associated with $0.016 \mathrm{X} 0.022$ " SS wires. Similarly, Abu-Shahba and Alassiry reported comparable labial crown torque during canine retraction using active and passive self-ligating brackets. In their study, the change in torque ranged between $0.97-1.27^{\circ}$. However, this may have been due to the positive bracket torque of $12^{\circ}$ and $15^{\circ}$ employed in their study. ${ }^{[16]}$

Despite the wide variability shown by the standard deviations of the means for the upper and lower canine torque, the histograms showed a gaussian distribution.

In our study, the canines were retracted over a 0.016 "X0.022" wire with a small negative torque in the bracket slot and no wire torsion. The engagement angle was probably insufficient to express the builtin bracket torque. This may have been adequate for our patients since the canines were not severely displaced buccally.

According to the recommendation, to overcome the play of the wire, in 0.022 " slot brackets, $18^{\circ}$ and $6^{\circ}$ engagement angle is required for 0.018 "X0.025" and 0.021"X0.025" stainless steel wires, respectively. It may be advisable to invert the brackets or incorporate positive wire torque in cases where there is a risk of root proximity to the labial cortical plates.
Most of the studies evaluating torque expression are in-vitro. ${ }^{[17,18]}$ The studies mostly evaluate the incisor rather than canine torque during retraction. Further trials are required to evaluate the bracketwire combinations for canine torque expression in a clinical setting.

\section{CONCLUSION}

Within the limitation of this single arm study, the canines were retracted into the extraction spaces with a labial crown inclination in the absence of positive built-in bracket torque.

\section{REFERENCES}

1. Zeitounlouian T, Zeno K, Brad B, Haddad R. Threedimensional evaluation of the effects of injectable platelet rich fibrin (i-PRF) on alveolar bone and root length during orthodontic treatment: a randomized split mouth trial. BMC Oral Health 2021; 21:92.

2. Schropp L, Wenzel A, Kostopoulos L, Karring T. Bone healing and soft tissue contour changes following singletooth extraction: a clinical and radiographic 12-month prospective study. Int $\mathrm{J}$ Periodontics Restorative Dent $2003 ; 23(4): 313-323$.

3. McLaughlin, R. P., Bennett, J. C., \& Trevisi, H. J. (2002). Systemized Orthodontic Treatment Mechanics. Mosby.

4. Sims AP, Waters NE, Birnie DJ.A comparison of the forces required to produce tooth movement ex vivo through three types of pre-adjusted brackets when subjected to determined tip or torque values. Br J Orthod. 1994; $21: 367-373$.

5. Hamdan A, Rock P. The effect of different combinations of tip and torque on archwire/bracket friction. Eur J Orthod. 2008; 30:508-514.

6. Clinical recommendations regarding use of cone beam computed tomography in orthodontics. Position statement by the American Academy of Oral and Maxillofacial Radiology. Oral Surg Oral Med Oral Pathol Oral Radiol. 2013; 116:238-257.

7. Aceton Group. X MIND trium User Manual. https://www. acteongroup.com/us/uploads/media/default/0001/01/96d7 f549b93beec4115e3e85e59b5e065c248c06.pdf accessed on July 25, 2018. P.35 
8. Puente M, Galban L, Cabo J. Initial stress differences between tipping and torque movements. A threedimensional finite element analysis. Eur J Orthod. 1996; 18: 329-339.

9. Beck BW, Harris EF. Apical root resorption in orthodontically treated subjects: analysis of edgewise and light wire mechanics. Am J Orthod Dentofac Orthop. 1994;105: 350-61.

10. Bennett J, McLaughlin R. Author's Notes. McLaughin and Bennet 5.0 with Forestadent. A bracket selection guide. FORESTADENT. 2019 https://cdn.starwebserver. se/shops/brotech/files/info_218_mclaughlinbennett_ bracketselectionguide_final_ansicht.pdf?_=1550668820. Accessed on May 19, 2020.

11. Harzer W, Bourauel C, Gmyrek H. Torque capacity of metal and polycarbonate brackets with and without a metal slot Eur J Orthod 2004; 26: 435-441.

12. Papageorgiou S, Sifakakis I, Doulis I, Eliades T, Bourauel C. Torque efficiency of square and rectangular archwires into 0.018 and 0.022 in. conventional brackets. Progress Orthod 2016; 17:5.
13. Archambault A, Lacoursiere R, Badawi H, Major P, Carey J, Flores-Mir C. Torque expression in stainless steel orthodontic brackets. A systematic review. Angle orthod. 2010; 80:201-210.

14. Roth R. The straight-wire appliance 17 years later. J Clin Orthod 1987; 21(9): 632-42.

15. Miethke RR. Third order tooth movements with straight wire appliances. Influence of vestibular tooth crown morphology in the vertical plane. J Orofac Orthop 1997; 58: 186-97.

16. Abu-Shahba R, Alassiry A. Comparative evaluation of the maxillary canine retraction rate and anchorage loss between two types of self-ligating brackets using sliding mechanics. J Orthod Sci 2019; 8:3.

17. Gioka C, Eliades T. Materials-induced variation in the torque expression of preadjusted appliances. Am J Orthod Dentofacial Orthop. 2004; 125:323-328.

18. Isaacson RJ, Lindauer SJ, Rubenstein LK. Moments with the edgewise appliance: incisor torque control. Am J Orthod Dentofacial Orthop. 1993; 103:428-438. 